九州大学学術情報リポジトリ

Kyushu University Institutional Repository

Über Den Gehalt An Trockensubstanz und Asche In Einem Bestimmten Volumen Gewebepulver Als Indizium Für Den Gehalt Des Pflanzenkörpers An Denselben Konst i tuenten

Koketsu, Riichiro

Botanischen Laboratorium, Kaiserlichen Kyushu Universität

https://doi.org/10.5109/22326

出版情報：九州大学大学院農学研究院紀要. 1 (3)，pp. 151-162，1924-12. Kyushu Imperial University バージョン：

権利関係 : 
Journal of the Department of Agriculture, Kyushu Imperial Úniversity, Vol. I, Nr.3

December 3I, I924

\title{
ÜBER DEN GEHALT AN TROCKENSUBSTANZ UND ASCHE IN EINEM BESTIMMTEN VOLUMEN GEWEBEPULVER AIS INDIZIUM FÜR DEN GEHALT DES PFLANZENKÖRPERS AN DENSELBEN KONSTITUENTEN ${ }^{1}$
}

\author{
Riichiro KôkErsu
}

Das Trockengewicht und der Aschengehalt eines Pflanzenkörpers werden, je nach den Umständen, in prozentischen oder in absoluten Werten angegeben. Bei den Blättern ะ. B. können wir den Stoffgehalt mit dem durchschnittlichen absoluten Gehalt in einem Blattindividuum, in einer Flächeneinheit, oder in einer Gewebevolumeinheit, oder auch durch den Gehalt in Prozenten des Gesamt- oder Trockengewichtes angeben $(2,3)$. Aber die Frage, welche Methode in einem gegebenen Falle die zweckmässigste sei, ist nicht leicht zu entscheiden. Selbst wenn ein Bestimmungsverfahren fehlerlos ausgeführt wird, so dürfte es doch häufig nicht möglich sein, eine biologisch zweckdienliche Deutung aus den Versuchsresultaten zu ziehen, da insbesondere bei lebenden Versuchsmaterialien, durch verschiedene Faktoren Schwankungen verursacht werden können. Es ist z. B. der Stoffgehalt in Prozenten des Gasaminewichtes von dem in der Mehrzahl der Fälle zu verschiedenen Zeiten schwankenden Wassergehalt der Materialien abhängig (4, 5).

Andererseits ist es aber auch schwer zu entscheiden, ob man in einem gegebenen Falle den Stoffgehalt durch Proz. des Frischgewichtes oder des Trockengewichtes beurteilen soll. Ja, es kommt wohl nicht selten vor, dass die Resultate einer quantitativen Bestimnung eines Stoffes, je nachdem man sie in Proz. des. Frischgewichtes oder des Trockengewichtes ausdrückt, sich geradezu umgekehrt verhalten (1). Um einen solchen Irrtum in der Beurteilung von Versuchsresultaten möglichst zu vermeiden, müsste man zunächst die inneren Bedingungen eines Versuchsmaterials feststellen, ehe man über die zu

I Arbeiten aus dem botanischen Labnratorium der Kaiserlichen Kyushu-Universität, No. 3. 
benützende Versuchsmethode entscheidet. Das ist aber sehr mühsam, u. U. sogar unmöglich.

Die Schwierigkeiten in cler Beurteilung des Stoffgehaltes eines Pflanzenkörpers könnten verringert werden, wenn man eine Versuchsmethode zur Anwendung bräuchte, die durch die Verschiedenheit der Bedingungen möglichst wenig beeinflusst wird. Theoretisch ist der Stoffgehalt in einer bestimmten Volumeinheit des Versuchsmaterials meist als gleich dem des ganzen Phanzenkörpers zu deuten. Aber die Bestimmung des Volumens eines Pflanzenkörpers ist in der Regel sehr schwierig. Dagegen aber kann, durch Messung des Volumens des aus dem Gewebe hergestellten Pulvers, eine volumetrische Angabe eines beliebigen Pflanzengewebes leicht anschaulich gemacht werden. Ich habe deshalb die nachstehenden Untersuchungen ausgeführt, um festzustellen, inwieweit die Bestimmung der in einer Volumeinheit des Gewebepulvers enthaltenen Stoffmenge für die Bestimmung des Trockengewichtes und des Aschengehaltes eines Pflanzenkörpers anwendbar ist.

Bevor ich aber die Ergebnisse meiner Versuche darlege, will ich hier die Versuchsmethodik kurz beschreiben. Das Gesamt- und das Trockengewicht des Materials wurde durch die gewöhnlichen Methoden bestimmt. Die Trocknung des Materials geschah im Trockenschrank bei $100-102^{\circ} \mathrm{C}$. Wenn das Versuchsmaterial durch Tau oder durch Waschen benetzt war, so wurde es mittels Fliesspapiers von dem Benetzungswasser befreit, bevor das Gesamtgewicht bestimmt wurde. Die Veraschung des Materials geschah einfach in Porzellantiegeln.

Zur Herstellung des Gewebepulvers wurde das im Trockenschrank wasserfrei gemachte Material in einem Mörser zermalmt, fein pulverisiert und durch $2 / 5 \mathrm{~mm}$ Sieb gesiebt. Zur Messung des Pulvervolumens wurdê immer ein kleiner Messzylinder von ca. $1 \mathrm{~cm}$ Durchmesser benützt.

Dic ganze Reihe der Bestimmungen wurde wie folgt nacheinander ausgefuhrt. Aus den Versuchsobjekten wurden 2 Serien von Materialgruppen nebeneinander gebildet und von jedèr Gruppe zuerst das Gesamtgewicht und dann das Trockengewicht bestimmt. Dann wiurde eine Serie verascht und der Aschengehalt bestimmt. Die noch übrige Serie wurde für die Herstellung des Gewebepulvers benützt. Die Bestimmung des Trockensubstanzgewichtes und des Aschengehaltes in einem bestimmten Voiunen des Pulvers ${ }^{2}$ wurde meistens dreimal seltens

2 Meistens $5 \mathrm{~cm}^{3}$, seltener $3 \mathrm{~cm}^{3}$; dann der Gehalt auf $1 \mathrm{~cm}^{3}$ umgerechnet. 
zweimal wiederholt um Durchschnittswerte zu erhalten, trotzdem die Resultate nur wenig voneinander abwichen.

Es scheint bei diesem Pulververfahren in der Messung des Volumens eine unvermeidliche Fehlerquelle zu liegen. Streng genommen ist eine fehlerlose Feststellung überhaupt nicht möglich. Aber nach meinen Erfahrungen sind die Abweichungen der Messungsresultate praktisch kaum merklich. Ich habe versucht den Fehler bei dem Einpacken des Materials dadurch zil verringern, dass ich den Messzylinder mit dem Material von einer bestimmten Höhe $(2 \mathrm{~cm})$ senkrecht auf den Tisch fallen liess und zwar so lange, bis die Verminderung des Volumens nicht mehr merklich war.

Vressucri I.-Von den auf ein und demselben Acker angebauten Glycine Soja Pflanzen wurden als Versuchsmaterial die Blätter ohne

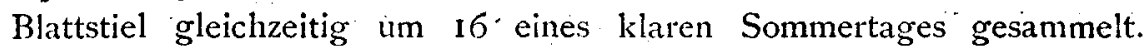
Sowohl bezügl. des Aschengehaltes als auch des Trockengewichtes wurden je drei Gruppen nebeneinander untersucht und zwar wurden ausser dem Prozentgehalt an Frish- und Trockensubstanz, auch der absolute Gehalt an Frisch- und Trockensubstanz in einer bestimmten Blattfächeneinheit," Gewebevolumeinheit ${ }^{4}$ und Pulvervolumeinheit bestimmt.

Von dem mittleren Fehler $\left(\sqrt{-\Sigma s^{2}}\right)$ zu urteilen, scheinen die Resultate aller hier benutzten Methoden mit gleicher Genauigkeit brauchbar zu sein (Tab. I). Die Abweichungen in den Resultaten waren die grössten bei der Bestimmung des Trockengewichtes in Proz. des Frischgewichtes, was wahrscheinlich durch die Verschiedenheit des Wassergehaltes im Material bedingt ist. Dagegen ist es merkwürdig, dass die Schwankungen bei den durch die Pulvermethode erhaltenen Resultaten in beiden Fällen die geringsten sind.

'T'aluelle r. Frgebnisse der Restimmungen an den gleichacitig gesammelten Blättern von Gljcine Soju.

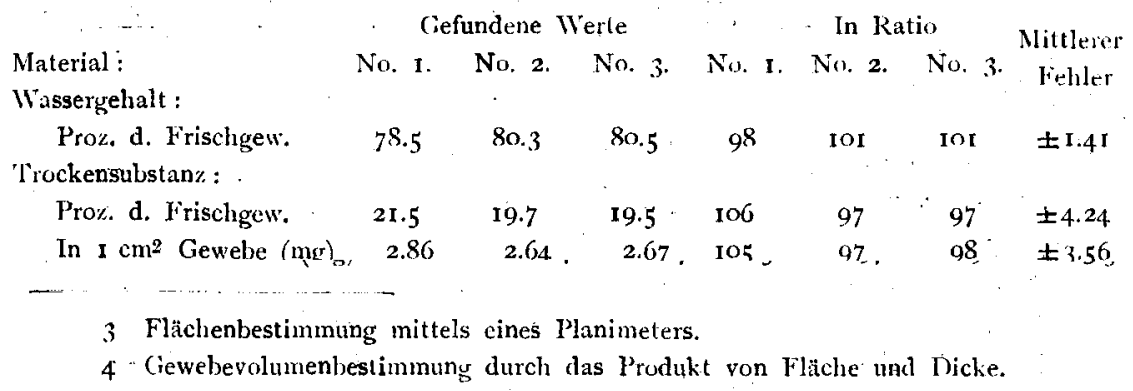




\begin{tabular}{|c|c|c|c|c|c|c|c|}
\hline . & \multicolumn{3}{|c|}{ Gefundene Werte } & \multicolumn{3}{|c|}{ In Ratio } & \multirow{3}{*}{$\begin{array}{c}\text { Mittlerer } \\
\text { Fehler }\end{array}$} \\
\hline Material : & No. 1. & No. 2. & No. 3 . & No. 1 & No. 2. & No. 3 . & \\
\hline \multicolumn{7}{|l|}{ Trockensubstanz: } & \\
\hline In $1 \mathrm{~cm}^{3}$ Gewebe (mg) & 140.6 & 135.2 & 132.6 & 103 & 99 & $9^{8}$ & \pm 2.16 \\
\hline In I cmi Pulver $(\mathrm{mg})$ & 400.0 & 417.0 & 416.0 & 97 & 102 & IOI & \pm 2.16 \\
\hline \multicolumn{8}{|l|}{ Aschensubstanz: } \\
\hline Proz. d. Frischgew. & I.9I & 1.83 & $1.8 \mathrm{I}$ & 103 & 99 & $9^{8}$ & \pm 2.16 \\
\hline Proz. d. Trockengew. & 9.45 & 8.89 & 8.79 & 105 & 98 & 97 & $\pm 3 \cdot 56$ \\
\hline In $1 \mathrm{~cm}^{2}$ Cewebe $(\mathrm{mg})$ & 0.27 & 0.25 & 0.26 & 104 & 96 & 100 & \pm 3.26 \\
\hline In I $\mathrm{cm}^{\approx}$ Gewelse (mg) & $\mathbf{1 3 . 4}$ & 12.4 & 12.9 & 104 & 96 & 100 & $-1=3.26$ \\
\hline In $1 \mathrm{~cm}^{3}$ Pulver (mg) & 34.8 & 36.0 & 36.7 & 97 & IoI & 102 & \pm 2.16 \\
\hline
\end{tabular}

Vkisuch 2.-An einem heissen Sommertage wurden drei Spezimen von Chenopodium album ausgewählt, davon die eine fast normal, eine andere welkend und eine andere noch stärker welkend waren. Auch hier diente nur die Blattspreite zu dem Versuch. Der Wassergehalt der Blätter der drei verschiedenen Pflanzen betrug $80.3 \%$, beziehw. $75.3 \%$ und $72.6 \%$, d.h. also ein Verhältnis von $106: 99: 95$ oder von 100:93:90 und das prozentuale Verhältnis des Trockengewichtes betrug $83: 103:$ I 14 oder $100: 124:$ I 38 . Natürlich ist diese grosse Abweichung des Trockengewichtes bei diesen drei Pflanzen nur scheinbar. In einem derartigen Falle bedeutet die Verschiedenheit des auf das Frischgewicht bezogenen prozentischen Gehaltes an Trockensubstanz nichts anderes als die Verschiedenheit des Wassergehaltes in dem Material.

Ganz anders dagegen stellten sich die Bestimmungsresultate bei unserer Pulvermethode; der Gehalt an Trockensubstanz in $1 \mathrm{~cm}^{3}$ Pulver betrug $567.0 \mathrm{mg}$ bezw. $565.2 \mathrm{mg}$ und $562.2 \mathrm{mg}$, in Ratio Ior : I00: 99 (Tab. 2).

Talelle 2. Resultate der Versuche mit verschiedenstark welkenclen Blättein von Chemopodimm allum, gegelsen in Ratio.

Material I. Material 2. Material 3. Abweichung

Wissergehalt : Fast normal Welkend Stärker welkend

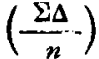

Proz. d. Frischgew

$$
106
$$

Tirnckengewicht :

Pro\%. d. Frischgew.

- In $1 \mathrm{~cm}^{3}$ Pulver

Aschengehalt :

Proz. d. Frischgew.

Proz. d. Trockengew.

In $1 \mathrm{~cm}^{3}$ Pulver

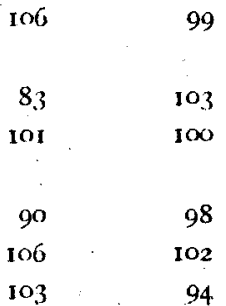

99

103

98

94
95

I 4

99

112

.92

103 $\pm 4.0$

$\pm 11.3$

$\pm 0.7$

$\pm 8.0$

$\pm 5.3$

ㄷ. 40 
Die Resultate der Bestimmung des Aschengehaltes verhielten sich, je nachdem sie auf Proz. des Frischgewichtes oder des Trockengewichtes bezogen wurden, gerade umgekehrt. Bezogen auf das Frischgewicht war der Aschengehalt un so grösser, und bezogen auf das Trockengewicht um so kleiner, je kleiner der Wassergehalt in dem Material war. Selbstverständlich ist der Aschengehalt in Proz. des Frischgewichtes durch die Verschiedenheit des Wassergehaltes nicht wenig beeinflusst. Andererseits ist die Tatsache, dass der Aschengehalt bezogen auf das Trockengewicht in welkenden Materialien kleiner ist, vielleicht auf Schwankungen des Assimilatgehalts in den Blättern zurückzuführen. Das Resultat der Pulvermethode aber ist theoretisch von solchen Bedingungen nur wenig abhängig. In der Tat waren, wie aus Tab. 2 zu ersehen ist, die Abweichungen nur gering.

Versuch 3.-Die Blätter von Glycine Soja, die auf ein und demselben Acker kultiviert waren, wurden zu verschiedenen Zeiten eines hellen Sommertages, d. h. um 5, teils um 10 und teils um I6 eingesammelt um das Trockengewicht und den Aschengehalt zu verschiedenen Zeiten zu vergleichen.

Der Gehalt an Trockensubstanz, der nebeneinander auf das Gesamtgewicht, auf eine Flächeneinheit und eine Pulvervolumeinheit bezogen, bestimmt wurde, war, wie erwarten stand, am grössten in den Blättern, die um 16 und am kleinsten in den Blättern, die um 5 eingesammelt worden waren. Die Abweichungen in dem Gehalt zu den verschiedenen Zeiten waren aber am grössten bei der auf das Frischgewicht sich beziehenden Bestimmung, was sicherlich in erster Linie auf die Verschiedenheit des Wassergehalts in den Materialien zurückzuführen ist (Tab. 3).

T'abelle 3. Resultate erhalten an den zu verschiedenen Tigeszeiten gesam. melten Blättern ron Gly'cine Sojar, gegeben in Ralio.

Wassergehalt :

Material Material Material Durchschnittl.

Proz. d. Frischgew. un 5. $\mathrm{um}$ IO. $\mathrm{um} 16$

Abweichung

Trockengewicht :

Proz. d. Frischgew.

104.99

\section{.97}

$\pm 2.7$

In $1 \mathrm{~cm}^{2}$ Gewebe

$\begin{array}{lr}\text { 86 } & \text { 10:3 } \\ 90 & \text { I0I } \\ 98, & 99\end{array}$

I I I

$\therefore 9.3$

In $1 \mathrm{~cm}^{3}$ Pulver

99

109

$\pm 6.7$

Aschengehalt :

Proz. d. Frischgew.

Proz. d. 'Trockengew.

91

In $1 \mathrm{~cm}^{2}$ Gewebe

In I cm3 Pulver

$100 \quad 100 \quad \pm 0,0$


1)agegen war der Aschengehalt am grössten in den um 5 gesammelten Blättern, wenn in Proz. auf das Trockengewicht bezogen, dagegen gerade umgekchrt um I6 am grössten, wenn auf das Gesamtgewicht bezogen. Im ersteren Falle dürfte diese Erscheinung auf Schwankungen in dem Assimilatgehalt und im letzteren Falle dürfte die scheinbar grössere Abweichung auf die Verschiedenheit im Wassergehalt zurückzuführen sein.

Theoretisch sollte die tägliche Schwankung in dem Aschengehalt nicht merklich sein, wie das ja auch experimentell schon nachgewiesen worden ist (6). Die zu erwartende Gleichmässigkeit in dem Aschengehalt der zu verschiedenen Tageszeiten gepfückten Blätter kam nur dann in Erscheinung, wenn der Gehalt in der Pulvervolumeinheit bestimmt wurde. Es ist also durch diesen Versuch erwiesen, dass die Aschengehaltsbestimmung $\mathrm{da}$, wo grosse Schwankungen in dem Trockengewicht oder dem Assimilatgehalt zu erwarten sind, nicht auf das Trockengewicht bezogen werden darf.

Versuch 4.-Aus den vorangehenden Versuchen ergibt sich, dass der Angabe des Gehaltes an Trockensubstanz oder Asche in Proz. nur wenig Bedeutung zukommt, wenn der Wasser- und Assimilatgehalt in den Versuchsmaterialien schwankt. Die Bestimmung des Gehaltes in der Flächeneinheit aber kann von diesen Bedingungen nur wenig beeinflusst sein. D. h. aber auch nur dann, wenn die Dicke der betr. Versuchsobjekte nicht wesentlich von einander abweicht. Um diese Tatsache sicherzustellen, habe ich den Trockensubstanz und Aschengehalt in den dimorphen Blättern von Eucalyptus globulus vergleichend bestinmt, deren Dicke sehr verschieden war. Die durchschnittliche Dicke der an älteren Zweigen hängenden Blätter betrug $393 \mu$ und bei Jungformblättern $269 \mu$; das bedeutet also ein Verhältnis von I I9: 8I oder I00:68. Auch der Wassergehalt wai ein stark abweichender und betrug $61.0 \%$ bezw. $66.6 \%$, d. h. also ein Verhältnis 96: 104.

Genau wie in den vorhergehenden Versuchen, wurde auch hier Trockensubstanz in Proz. bezogen auf das Frischgewicht, in der Flächeneinheit der Blattspreite, in der Volumeinheit des"Blattgewebes und in der Volumeinheit des Gewebepulvers festgestellt. Die Resultate deuteten stets darauf hin, dass der Gehalt an Trockensubstanz in den Jungformblättern kleiner als in den Altformblättern war. Dabei wurde die grösste Abweichung in den Resultaten gewonnen bei der Bestimmung der Trockensubstanz in der Flächeneinheit gefunden. Das ist fraglos clurch die Verschiedenheit in der Blattdicke verursacht (Tab. 4). 
Tabelle 4. Versuchsresultate erhalten an den Blättern von Eucalyptus globulus, gegeben in Ratio.

\begin{tabular}{|c|c|c|c|}
\hline & Altformblätter & Jungformblätter & $\begin{array}{l}\text { Dürchischinitti } \\
\text { Abweichung }\end{array}$ \\
\hline Blattdicke: & 119 & $S_{\mathbf{I}}$ & \pm 19 \\
\hline \multicolumn{4}{|l|}{ Wassergehalt : } \\
\hline Proz. d. Frischgew. & 96 & 104 & \pm 4 \\
\hline Trockengewicht: & & & . \\
\hline Proz. d. Frischgew. & 108 & 92 & \pm 8 \\
\hline In $1 \mathrm{~cm}^{2}$ Gewebe & 132 & 68 & $\pm 3^{2}$ \\
\hline In $1 \mathrm{~cm}^{3}$ Gewebe & Ir3 & 87 & \pm 13 \\
\hline In $\mathrm{I} \mathrm{cm}^{3}$ Pulver & 106 & 94 & \pm 6 \\
\hline \multicolumn{4}{|l|}{ Aschengehalt: } \\
\hline Proz. d. Frischgew. & 88 & I 12 & $\therefore \mathbf{I} 2$ \\
\hline Proz. d. Trockengew. & 80 & I 20 & \pm 20 \\
\hline In $1 \mathrm{~cm}^{2}$ Gewebe & 107 & 93 & \pm 7 \\
\hline In $1 \mathrm{~cm}^{3}$ Gewebe & 92 & 108 & \pm 8 \\
\hline In $1 \mathrm{im}^{3}$ Pulver & 84 & 116 & $\pm \mathbf{I} 6$ \\
\hline
\end{tabular}

Auch bezüglich des Aschengehalts ist der grössere Wert für die Altformblätter bei der Bestimmung des Gehaltes auf die Flächeneinheit zweifellos nur auf den Unterschied in der Dicke zurückzuführen. Es zeigt sich also aus diesem Versuche, dass bei grossen Unterschieden in der Blattdicke, die Bestimmung auf die Flächeneinheit nicht zulässig ist.

Versucr 5--Es gibt viele Blätter, deren Fläche oder Volumen nicht leicht zu bestimmen ist. Bei solchem Material ist die Bestimmuug des Stoffgehaltes in den Blättern nur durch prozentische Angabe ratsam. Wird aber der absolute Gehalt in einer bestimmten Materialeinheit gewünscht, so ist unsere Methode den Gehalt in einem bestimmten Pulvervolumen zu bestimmten, zu empfehlen.

Als Beispiel für einen solchen Fall wurde der Gehalt an Trockensubstanz und Asche in den linearen Blättern von Pinus Thunbergii bestimmt. Drei Materialgruppen, die zu drei verschiedenen Tageszeiten (um 5, Io und 16) eines heissen, aber trüben Sommertages gepflückt wurden, wurden nebeneinander untersucht. Hier stand zu erwarten, dass die Verschiedenheit des Gehaltes an Wasser und Assimilaten die prozentischen Wert für den Stoffgehalt beeinflussen würden, wenn auch die Beeinflussung unter den gegebenen Versuchsbedingungen nicht sehr gross sein würde. Die Resultate bestätigten die Erwartung (Tab. 5), ausserdem bestätigten sie weiter die Brauchbarkeit der Pulvermethode. 
'abelle 5. Resultate gewonnen an den Blättern von Pinus Thunbergäi, gegelsen in Ratio.

Wassergehalt :

$\begin{array}{cccc}\text { Material } & \text { Material } & \text { Material } & \text { Durchschnittl. } \\ \text { um 5. } & \text { um ro. } & \text { um I6. } & \text { Abweichung }\end{array}$

Proz. d. Irischgew. . IOI

$\begin{array}{llll}\text { IOI } & \text { IOO } & 99 & \text { 土0.7 }\end{array}$

Trockengewicht :

Proz. d. Frischgew.

In I $\mathrm{cm}^{3}$ Pulver 9

$97 \quad$ I00 $\quad 103 \quad \pm 2.0$

Aschengehalt :

Proz. d. Frischgew.

Proz. d. Trockengew.

In $1 \mathrm{~cm}^{3}$ Pulver

98 IOI IOI 11.3

Versuch 6.-Zweck dieses Versuches war es die Brauchbarkeit der Pulvermethode auch an anderen Materialien $z u$ beweisen. Es wurde hier der Gehalt an Trocken- und Aschensubstanz in einjährigen und dreijährigen Stengeln von Morus alba verglichen. Ilier kanı natürlich nicht der Gehalt in der Gewebeflächeneinheit und auch kaum der Gehalt in der Gewebevolumeinheit bestimmt werden. $\mathrm{Da}$ hier der Wassergehalt $(\mathbf{7 2 . 9} \%$ in dem einjährigen und $45.9 \%$ in dem dreijährigen Stengel) ein so verschiedener war, so war ein bedeutender Fehler in der prozentischen Bestimmung des Trockengewichtes zu erwarten (Tab. 6). Andererseits wurde eine starke Abweichung auch in der Bestimmung des Aschengehaltes in Proz. der Trockensubstan\% gefunden. Das ist, teilweise wenigstens, auf die Verschiedenheit der chemischen und physikalischen Beschaffenheit der Trockensubstanz zurückzuführen. Theoretisch sollte die Fehlerquelle bei der Bestimmung des Stoffgehaltes in der Pulvervolumeinheit am kleinsten sein. Das Ergebniss der Versuche deutet darauf hin.

Tabelle 6. Resiltate gewonnen an den Stengeln von Morus alba, gegeben in Ratio.

Wassergehalt :

$\begin{array}{rcc}\begin{array}{c}\text { Einjähriger } \\ \text { Stengel }\end{array} & \begin{array}{c}\text { Dreijähriger } \\ \text { Stengel }\end{array} & \begin{array}{c}\text { Durchschnittl. } \\ \text { Abweichung }\end{array} \\ 123 & 77 & \pm 23 \\ 67 & 133 & \pm 33 \\ 104 & 96 & \pm 4 . . \\ 131 & 69 & \pm 31 \\ 158 & 42 & \pm 5^{8} \\ 141 & 59 & \pm 41\end{array}$

Versuch 7.-Aus der vorhergehenden Versuchen ging hervor, dass eine rationelle Bestimmung des Trockensubstanz- und Aschengehaltes eines Pflanzenkörpers in vielen Fällen durch eine der gewöhnlich 
gebräuchlichen Methode nicht möglich ist. Es ist also die Anwendbarkeit einer der gewöhnlichen Methoden auf solche Fälle beschränkt, in denen sie dafür geeignet ist. Unsere Pulvermethode aber hat ein sehr weites Anwendungsgebiet.

$\therefore$ So ist z. B. wenn die Fragestellung auf den Stoffgehalt in verschiedenen Pflanzenteilen gerichtet ist, kann nur unsere Methode ohne merkliche Fehlerquellen angewendet werden. Ich habe dazu Versuche an Blättern, Stengeln und Wurzeln von Celocia cristata und Impatiens Balsamina ausgeführt.

Der prozentuale Wassergehalt war sowohl bei Cilocia, als auch bei Impaticus am grössten in Stengel und am geringsten in den Blättern. Die Beschaffenheit der Trockensubstanz wird dabei in den verschiedenen Organen nicht wenig verschieden sein. Es ist daher in diesem Falle zú erwarten, dass der prozentuale Gehalt án : Trockensubstanz und Asche durch diese Verschiedenheit in nicht geringem Masse beeinflusst werde. Daher mag es kommen, dass die Resultate der Versuche mit den verschiedenen Methoden nicht übereinstimmen (Tab. 7 u. 8).

'Tabelle 7. Resultate der Bestimmungen an verschiedenen Organen von Celacia cristata, gegeben in Ratio.

\begin{tabular}{|c|c|c|c|c|}
\hline & Blatt & Stengel & Wurzel & $\begin{array}{l}\text { Turchschnittl. } \\
\text { Abweichung }\end{array}$ \\
\hline Wassergehalt : & & & . & \\
\hline Proz. d. Friscligew. & 98 & 102 & 100 & $\pm \mathrm{x} \cdot 3$ \\
\hline 'lrockengewicht: & & & & \\
\hline Proz. d. Frischgew. & 129 & $7 \cdot 1$ & 100 & \pm 19.3 \\
\hline In $1 \mathrm{~cm}^{3}$ Pulver & 107 & 99 & 94 & \pm 4.7 \\
\hline Aschengehalt : & & & & \\
\hline Proz. d. Frischgew. & 121 & 89 & 90 & \pm 14.0 \\
\hline Proz, d. T'rockengew. & 93 & 121 & 86 & \pm 14.0 \\
\hline In $1 \mathrm{~cm}^{\mathbf{3}}$ Pulver & 106 & 116 & 78 & \pm 14.7 \\
\hline
\end{tabular}

Tebelle 8. Resultate der Bestimmungen an verschiedenen Organen von Impatiens Balsamina, gegeben in Ratio.

\begin{tabular}{|c|c|c|c|c|c|}
\hline$\therefore$ & & Blatt & Stengel & Wurzel & $\begin{array}{l}\text { Durchschnittl } \\
\text { Abweichung }\end{array}$ \\
\hline Wassergehalt : & & & & & \\
\hline Proz. d, Frischgew. & & 97 & 102 & IOI & \pm 2.0 \\
\hline 'l'rockengewicht : & & & & & \\
\hline Proz. d. Frischgew. & $\cdot$ & 160 & 67 & 73 & \pm 40.0 \\
\hline In $1 \mathrm{~cm}^{\mathrm{s}}$ Pulver & & 131 & 93 & 76 & \pm 20.7 \\
\hline Aschengehalt : & & . & & & \\
\hline Proz. d. Frischgew. & & 120 & 97 & 83 & $\pm 13 \cdot 3$ \\
\hline I'roz. d. 'Trockengew. & & 64 & 127 & Ioy & \pm 24.0 \\
\hline In I $\mathrm{cm}^{3}$ Pulver & & 94 & I 23 & 83 & $\pm 15 \cdot 3$ \\
\hline
\end{tabular}


Daraus ergibt sich ein zu hoher prozentischer Wert für das Trockengewicht der Blätter und ein zu kleiner in dem der Stengel, was fraglos auf die Verschiedenheit in dem Wassergehalt zurückzuführen ist. Auf das Gesamtgewicht bezogen war der prozentuale Aschengehalt zu hoch beim Blatt, auf das Trockengewicht bezogen war es dagegen zu klein beim Blatt und zu gross bei der Wurzel, was vielleicht durch Schwankungen in dem Wassergehalt bezw. in den Assimilaten zurückzuführen ist. Auch hier erwies sich unsere Methode wieder als recht brauchbar.

Vkissucr 8.-Ich führte hier einen Versuch mit den Blättern verschiedener Spezimen der Oenothera biennis, die an verschiedenen Standorten, teils auf einem sonnigen Strande, teils auf schattigem Inlande gewachsen waren, aus. Der Wassergehalt betrug bei den ersteren $80.5 \%$ und bei den letzteren $89.5 \%$. So trat der Einfluss der Verschiedenheit des Wassergehaltes auf den Stoffgehalt in Proz. bezogen auf das Frischgewicht deutlich hervor (Tab. 9). Andererseits wurde ein $7 u$ grosser Wert für das auf das Trockengewicht bezogene prozentuale Aschengehalt, wie zu erwarten stand, in den Blättern der Schattenpflanzen gefunden.

Tabelle 9. Resultate der bestimmungen an den Blättern von an verschiedenen Orten wachsender O 'nothera birmis, gegeben in Ratio.

\begin{tabular}{|c|c|c|c|}
\hline 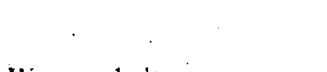 & Schattenpflanzen & Sunnenptlan\%en & $\begin{array}{l}\text { Durchschnittl } \\
\text { Abweichung }\end{array}$ \\
\hline Wassergehalt: & & & \\
\hline Proz. d. Frischgew. & 105 & 95 & \pm 5 \\
\hline 'Trockensubstanz: & & & $\therefore \quad$ \\
\hline Proz. d. lrischgew. & 70 & 130 & \pm 30 \\
\hline In $1 \mathrm{~cm}^{3}$ I'ulver & 93 & 107 & \pm 7 \\
\hline Aschengehalt : & & & \\
\hline Proz. d. lirischgew. & 77 & 123 & \pm 23 \\
\hline Proz. d. Trockengew. & $-\mathbf{I I} \mathbf{1}$ & 89 & $\pm \mathrm{II}$ \\
\hline In $1 \operatorname{cin}^{3}$ Pulver & IO2 & 98 & \pm 2 \\
\hline
\end{tabular}

Virsuch 9.-Bekanntlich besitzen verschiedene Pflanzen, obwohl sie auf demselben Boden gewachsen sind, verschiedenen Gehalt an Trockènsubstanz und Mineralstoffen. Ein rationeller, zahlenmässiger Verglêich des Stoffgehaltes verschiedener Pflanzen ist aber mit den durch die gewöhnliche proz. Bestimmungsmethode erhaltenen Werten schwer anzustellen, da die Beschaffenheit der verschiedenen Pflanzen in der Regel sehr verschieden ist. Hier ist ebenfalls unsere Pulvermethode sehr zẅeckmässig.

Ich habe ein vergleichende Bestimmung des Gehaltes an Trocken- 
substanz und Asche an drei verschiedenen Gewächsen, Acalypha ausiralis, Bulbostylis barbuta und Portulaca oleracea, die an einem Platze nahe einander aufgewachsen waren. gemacht. Die erstgenannte besitzt einnen verholzten Stengel, die zweite ist eine Graminee und die dritte eine halbsukkulente, es ist also ihre stoffliche Beschaffenheit ziemlich verschieden.

Als Versuchsmaterial diente der ganze Pflanzenkörper, samt Blättern, Stengeln und Wurzeln. Wie zu erwarten zeigte der Wassergehalt eine grosse Schwankung, und zwar erhielt ich den grössten Wert für Portulaca und den kleinsten für Acalypha. Der Vergleich des Trockensubstanzgehaltes in Proz. des Gesamtgewichtes hat also wenig mehr Bedeutung als eine Verschiedenheit des Wassergehaltes zu zeigen.

Der Wert für den Aschengehalt in Proz. des Trockengewichts stimmte mit dem durch die Pulvermethode erhaltenen ziemlich gut überein, obgleich zu vermuten ist, dass der erstere nicht wenig durch die Verschiedenheit der Trockensubstanz in den verschiedenen Pflanzen beeinflusst wird (Tab. Io).

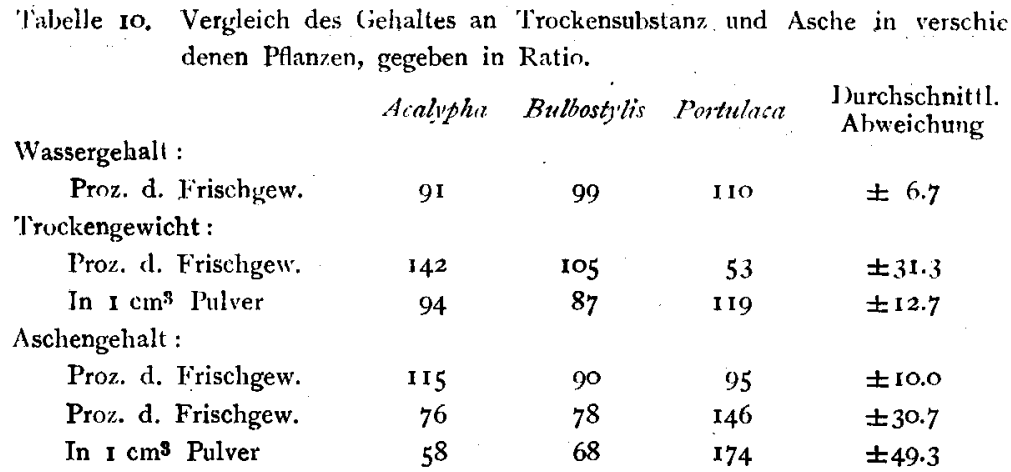

Die Zweckmässigkeit der Pulvermethode für die Bestimmung des Trockensubstanzgewichtes und des Aschengehaltes im Pflanzenkörpers unter verschiedenen Versuchsbedingungen ergibt sich aus obigen Versuchen. Insbesondere ist diese Methode da anwendbar, wo die zu untersuchenden Materialien in ihrer stofflichen Zusammensetzung weit voneinander abweichen. In dieser Hinsicht hat diese Methode ein grosses Anwendungsgebiet, sowohl in der Physiologie als auch in der Oekologie. 
Es ist weiterhin diese Methode vermutlich mit gleich gutem Erfolge für die Bestimmung des Wassergehalteś im Pṭanzenkörper anzuwenden, wenn man nämlich das ganze Volumen des von dem zu untersuchenden Material hergestellten Pulvers feststellt und dann Wassergehalt auf ein bestimmtes Volumen des Gewebepulvers berechnet.

botanisches Laloratorium, Kaiserliche Kyushu-Lniversitat

Oktobei 1924

\section{LITERATUK}

I) CulbNal, A. Ch., Diumal variations in the total nitrogen content of foliage leaves. Ann. Bot. 39, $511-518,1923$.

2) CzApek, F., Biochemie der Pflanzen. Bd. II. 2. Aufl. Jena 1920.

3) Grafe, G., Ernährungsphysiologisches Praktikum höherer Pflanzen. Berlin 1914.

4) KNIGHT, R. C., Further observations on the transpiration, stomata, leaf water-content and wirting of plants. $\Lambda$ nn. Bot. 36, 361-381, r922.

5) Iivingston, B. E. and W. H. Brown, Relation of daily march of transpiration to variation in the water-content of foliage leaves. Bot. Gaz. 53, 309-330, 1912.

6) RAMANN, E., Mineralstoffwechsel von Baumblättern zur Tages- und Nachtzeit. Jahrb. f. wiss. 'Bot. 50, 84-9̀I, 1912. 